\title{
Optimising graft survival in endothelial keratoplasty for endothelial failure secondary to cytomegalovirus endotheliitis
}

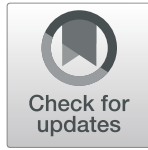

Milton C. Chew ${ }^{1}$, Donald T. Tan ${ }^{1,2}$, Soon-Phaik Chee ${ }^{1,2,3,4}$ and Lim Li ${ }^{1,2,4^{*}}$

\begin{abstract}
Background: There is limited information regarding Descemet stripping automated endothelial keratoplasty (DSAEK) for endothelial failure secondary to cytomegalovirus (CMV) endotheliitis. Treatment is difficult with high recurrence rates. We describe a case when systemic valganciclovir therapy is directed by aqueous CMV-DNA levels, leading to good graft survival.

Findings: A 59-year-old male with bilateral CMV endotheliitis despite antiviral therapy developed endothelial failure and underwent DSAEK. Prior to surgery, aqueous polymerase chain reaction (PCR) for CMV was repeatedly performed, where CMV-positive episodes were treated with systemic valganciclovir. Monthly aqueous analysis was performed until CMV-DNA was undetectable before DSAEK was performed. Post-operative prophylactic systemic valganciclovir treatment was instituted and switched to topical valganciclovir treatment when aqueous samples were negative for CMV.

Conclusion: Targeted aqueous sampling for CMV-DNA perioperatively guides antiviral therapy and ensures adequacy of treatment, minimising the duration of systemic valganciclovir therapy to reduce adverse effects of long-term treatment.
\end{abstract}

Keywords: Cytomegalovirus endotheliitis, Endothelial failure, Descemet stripping automated endothelial keratoplasty, Aqueous polymerase chain reaction

\section{Introduction}

Corneal endotheliitis is a clinical entity characterised by the presence of keratic precipitates (KPs), corneal edema and mild iritis [1]. Cytomegalovirus (CMV) infection has been shown to be an important cause of corneal endotheliitis [2,3]. If left untreated, it can lead to permanent endothelial damage requiring corneal transplantation. In cases where endothelial keratoplasty has been subsequently performed, recurrence is common despite anti-CMV treatment [4].

In this report, we present a case of bilateral endothelial failure secondary to recurrent CMV endotheliitis that subsequently underwent Descemet stripping automated endothelial keratoplasty (DSAEK). Treatment

\footnotetext{
* Correspondence: lim.li@singhealth.com.sg

${ }^{1}$ Singapore National Eye Centre, 11 Third Hospital Ave, Singapore, Singapore 168751, Singapore

${ }^{2}$ Singapore Eye Research Institute, Singapore, Singapore

Full list of author information is available at the end of the article
}

with anti-CMV therapy during recurrence optimised graft survival.

\section{Case report}

A 59-year-old gentleman presented in May 2008 for persistent corneal edema, with a history of bilateral recurrent anterior hypertensive uveitis. He had no significant past medical history, nor a history of immunological or infectious diseases of note. He did not have any systemic immunosuppression or significant oncological history. On examination, the bestcorrected visual acuity (BCVA) was $6 / 12$ in the right eye (RE) and $6 / 9$ in the left eye (LE). There was right corneal edema with multiple fine coin-shaped keratic precipitates (KPs). His left eye had few KPs (Fig. 1a-d). The right intraocular pressure (IOP) was raised at $35 \mathrm{mmHg}$, despite being on 3 topical glaucoma medications, and the left was $18 \mathrm{mmHg}$. Posterior segment examination was unremarkable. 


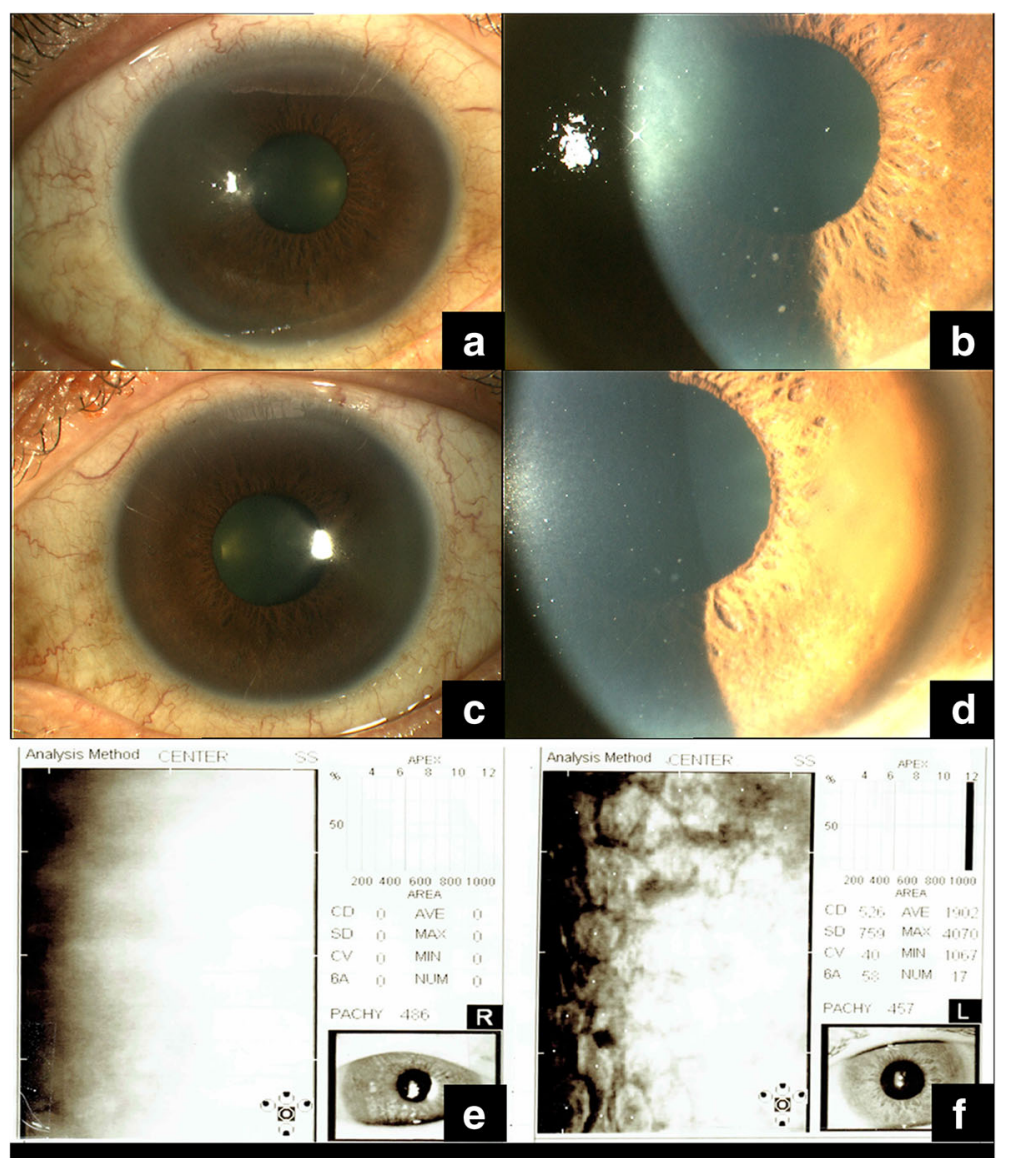

Fig. 1 Slit-lamp photographs on initial presentation. Sectorial corneal edema temporally with fine coin-shaped keratic precipitates in the right eye. $(\mathbf{a}, \mathbf{b})$ The left eye with a clear cornea but with similar fine coin-shaped keratic precipitates. (c, $\mathbf{d})$ The endothelial cell count of both eyes at initial presentation $(\mathbf{e}, \mathbf{f})$

Endothelial cell count was unattainable due to corneal edema in his right eye, and 526 cells $/ \mathrm{mm}^{2}$ in his left eye (Fig. 1e, f).

Aqueous tap was positive for CMV but negative for herpes simplex and varicella zoster virus in both eyes on qualitative polymerase chain reaction (PCR). Quantitative CMV PCR revealed a viral load of $6.3 \times 10^{4}$ copies/ $\mathrm{ml}(\mathrm{RE})$ and $9.3 \times 10^{5}$ copies/ml (LE). He was co-managed with an infectious disease consultant and treated based on our centre's treatment regime of oral valganciclovir $900 \mathrm{mg}$ twice daily for 6 weeks, followed by 450 $\mathrm{mg}$ twice daily for a further 6 weeks. Topical ganciclovir ophthalmic gel $0.15 \%$ (Virgan; Laboratories Théa, Clermount-Ferrand, France) 5 times a day was added. Whilst on systemic therapy, he underwent monthly aqueous taps, which showed decremental CMV-DNA titres. Subsequently, systemic treatment was stopped after 4 months when both eyes became CMV negative.

As the right IOP was poorly controlled despite maximum glaucoma medications, he underwent Ahmed tube implantation on May 2008 and subsequently right trabeculectomy on March 2009. His left eye also developed secondary glaucoma and trabeculectomy was performed in November 2008. Thereafter, his IOPs came under good control.

By August 2009, he had developed bilateral corneal endothelial failure (Fig. 2a, b). His BCVA dropped to 6/ 21 in the right eye and 6/15 in the left eye. Pre-operative central corneal thickness was $777 \mu \mathrm{m}$ in the right eye and $626 \mu \mathrm{m}$ in the left eye. As the eyes remained quiescent and were CMV negative, and the IOPs were well controlled, DSAEK was offered. He was given CMV prophylaxis with valganciclovir $900 \mathrm{mg}$ twice daily for 3 days pre-operatively, continued for 3 weeks post-operatively whilst the patient was on intensive post-operative topical corticosteroids. The corticosteroid regime consisted of topical prednisolone acetate $1 \% 3$ hourly for 3 weeks, followed by gradual tapering over time. Topical ganciclovir $0.15 \%$ ophthalmic gel was also prescribed pre and post-operatively. He successfully underwent right DSAEK on October 2009 and left DSAEK on January 2010. He recovered uneventfully with bilateral clear 


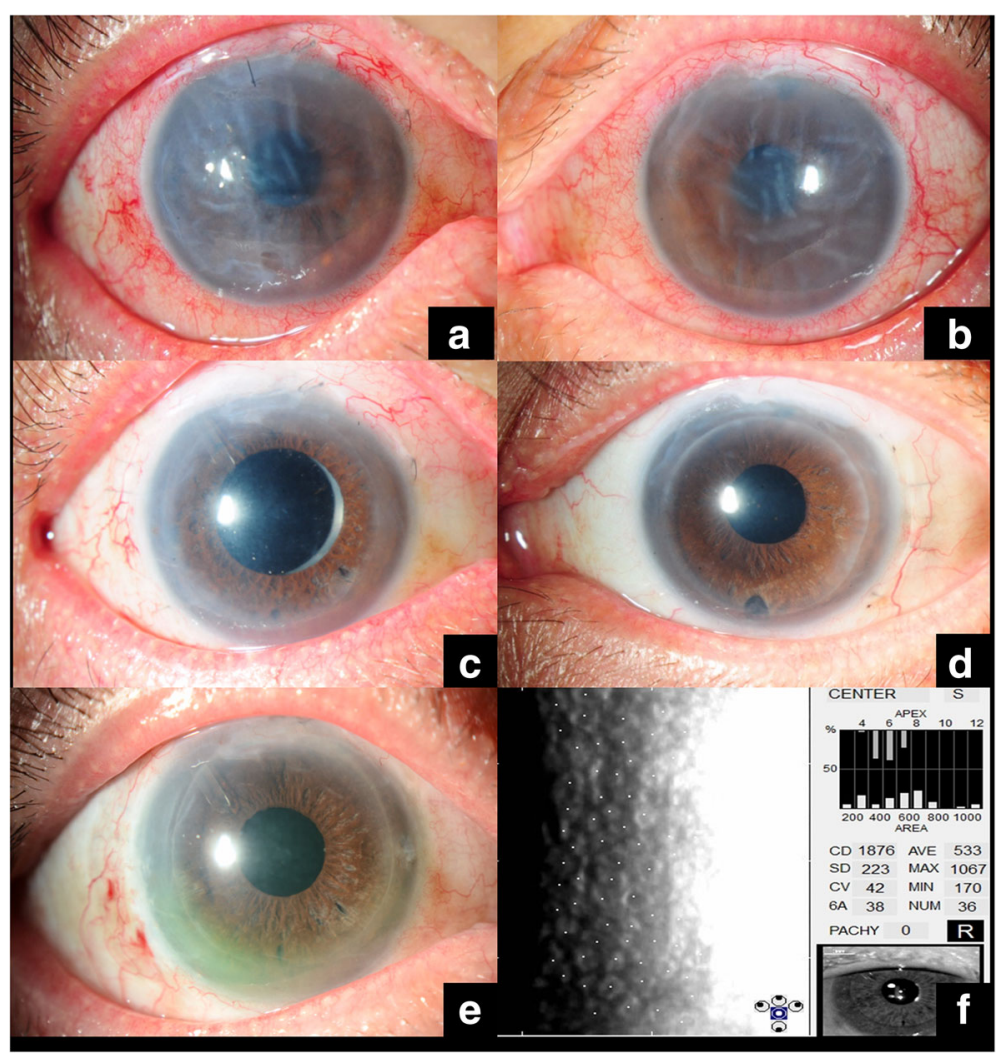

Fig. 2 Endothelial failure and corneal decompensation in the right (a) and left (b) eyes. Clear grafts in the right (c) and left (d) eyes after successful DSAEK surgery. Clear graft in the right eye (e) and latest endothelial cell count (f)

DSAEK grafts and BCVA of $6 / 7.5$ on the right (Fig. 2c) and $6 / 6$ on the left (Fig. 2d).

At the 2-month post-operatively mark, we performed a routine screening aqueous tap for both eyes. Aqueous CMV-DNA titres demonstrated viral load of $1.4 \times 10^{4}$ copies/ml (RE) and non-detectable (LE) respectively. In view of the recurrence in his right eye, systemic valganciclovir was restarted. Monthly aqueous taps showed decremental CMV titres and were negative for CMVDNA after completion of 4 months of systemic treatment. He was hence switched to long-term prophylactic topical ganciclovir. He maintained a clear DSAEK graft in both eyes with no recurrence for 3 years with good visual acuity of 6/7.5 in the right and 6/6 in the left.

On January 2014, his right eye developed CMV recurrence. He presented with blurred of vision, with visual acuity of $6 / 21$ in the right eye, localised corneal edema and KPs. IOP was normal. A diagnostic aqueous tap that was performed and showed CMV-DNA viral titres of $110,820 \mathrm{copies} / \mathrm{ml}$. He was restarted on systemic valganciclovir, with a monthly repeated aqueous tap to assess the viral load. After 3 months of treatment by April 2014, the corneal edema had resolved and the graft was clear. Repeat aqueous CMV-DNA was negative. The patient was then switched back to long-term prophylactic topical ganciclovir gel. He continued to have good vision and remained asymptomatic for another year. By May 2015, his right eye vision progressively worsened and eventually developed late graft failure from late endothelial failure, central corneal thickness of $683 \mu \mathrm{m}$ and normal IOP with a negative aqueous CMV-DNA. He subsequently underwent a repeat right DSAEK on September 2016 with the similar pre-operative and post-operative anti-CMV regime as previously described. On latest review on March 2019 , at 2.5 years post-operatively, his BCVA is $6 / 9$ with a clear graft and a good endothelial cell count of 1876cells $/ \mathrm{mm}^{2}$ (Fig. 2e, f).

His left eye had good vision for 4 years with BCVA of 6/6 after undergoing DSAEK in January 2010. He developed late endothelial failure and corneal decompensation with negative aqueous CMV-DNA by March 2014 . He underwent a repeat left DSAEK on September 2014 with the similar pre-operative and post-operative antiCMV regime as described. However, he did not continue with post-operative systemic valganciclovir due to financial constraints. Subsequently, he developed CMV recurrence with KPs and graft edema, normal IOP and positive aqueous CMV-DNA on January 2016, with subsequent graft failure by September 2016. He had a repeat 
DSAEK performed on May 2018 with a pre-operative negative aqueous CMV-DNA tap and underwent the similar anti-CMV oral valganciclovir regime. Post-operatively, he recovered uneventfully and IOP was normal. On the latest review on March 2019, his BCVA was 6/9 with a clear graft. As of the latest review, his current medications are topical dexamethasone $0.1 \%$ once a day to both eyes.

\section{Discussion}

Cytomegalovirus infection is an important cause of corneal endotheliitis. Seroprevalence ranges from 45$100 \%$ [5] and is higher in Asian countries [6]. They usually present unilaterally, but bilateral involvement has been reported [1, 7]. Clinical manifestations include coin-shaped keratic precipitates in a linear pattern, hypertensive anterior uveitis, and progressive endotheliitis (Fig. 1a-d) [2, 3]. This commonly leads to a presumptive misdiagnosis of herpetic keratouveitis [8], with poor response to anti-herpetic treatment. Prompt recognition and treatment with anti-CMV therapy are essential to prevent progressive endothelial deterioration [9].

This case report illustrates that whilst endothelial failure secondary to CMV endotheliitis is an important consideration, endothelial keratoplasty can still be performed with good outcome. We recommend preoperative aqueous PCR analysis for CMV-DNA prior to planning for corneal transplantation. Patients who are CMV-positive should have systemic valganciclovir initiated. In our centre, the treatment regime for CMV-positive endotheliitis consists of oral valganciclovir $900 \mathrm{mg}$ twice daily for 6 weeks followed by 900 $\mathrm{mg}$ once daily for a further 6 weeks [2]. Topical ganciclovir ophthalmic gel is also prescribed 5 times a day $[4,10]$. After completing the treatment regime, monthly repeated aqueous taps should be performed to monitor the decremental quantitative CMV-DNA titres. Only when aqueous CMV titres are negative we can stop the systemic treatment and proceed with DSAEK. Post-operatively, our centre recommends 3 weeks of continued prophylactic systemic valganciclovir therapy whilst the patient is on intensive topical corticosteroids to prevent CMV reactivation. As with herpes simplex, which is also associated with reactivation following the stress of surgery, oral antivirals post-operatively help to prevent recurrence [11]. Thereafter, we stop systemic anti-viral therapy too but continue long-term topical ganciclovir therapy to reduce recurrence. We also routinely perform a repeated aqueous sampling at the 2-month post-operative mark to ensure no recurrence.

To date, there is no consensus for the optimal treatment duration for CMV endotheliitis. Systemic valganciclovir treatment has a good response rate but is associated with a high recurrence rate [12]. The recurrence usually occurs after discontinuing of antiviral medications [4]. Furthermore, long-term systemic valganciclovir therapy can lead to serious adverse effects including pancytopenia and myelosuppression [13]. Topical ganciclovir gel has be associated with a lower recurrence rate compared to systemic treatment [12]. However, whilst topical ganciclovir reduces the frequency of recurrence, it was not associated with prolonging the time to recurrence [14]. Therefore, we recommend management to be directed by CMVDNA aqueous sampling as described above. This way, the duration of systemic ganciclovir can be reduced to minimise side effects, yet with the confidence that the CMV endotheliitis has been adequately treated. Despite anterior chamber paracentesis and sampling being a valuable diagnostic tool, there are concerns regarding rare but potential serious complications of endophthalmitis [15] and trauma to iris and lens. However, it has been shown to be a safe procedure in the hands of an experienced ophthalmologist with adequate aseptic precautions [16, 17].

Whilst we acknowledge the limitations of a case report, we believe that this case highlights an important diagnostic approach in the management of CMV endotheliitis in post-corneal transplant. Corneal endotheliitis is a clinical diagnosis, and a high index of suspicion is warranted when a patient presents with the coin-shaped KPs typically arranged in a linear pattern associated with hypertensive uveitis and localised corneal edema refractory to corticosteroid treatment [2, 3]. However, for patients who have undergone corneal transplantation, it can be difficult to distinguish between CMV reactivation and graft rejection. Inappropriate treatment with corticosteroids may exacerbate the CMV viral infection, increasing inflammation and endothelial damage with subsequent graft failure [10]. Therefore, timely aqueous CMV-DNA analysis can avoid the diagnostic dilemma. In our case, the patient presented with right eye graft edema and keratic precipitates in January 2014. Aqueous sampling confirmed CMV reactivation, and that enabled appropriate systemic antiviral treatment to be initiated early to prevent further endothelial damage and graft failure. The corneal edema resolved with anti-viral treatment and the patient maintained a clear graft with good vision. In contrast, when there was a recurrence of CMV endotheliitis in his left eye in January 2016, his financial constraints and resultant lack of appropriate systemic anti-viral therapy led to irreversible graft failure. The different outcomes between the 2 eyes highlight the importance of instituting early systemic 
anti-viral therapy in recurrent CMV infection to prevent graft failure.

Late endothelial failure and graft failure eventually occurred in our patient in both eyes despite our best efforts. This highlights the difficulty of management of recurrent CMV endotheliitis post-DSAEK. We advocate firstly the optimisation and control of intraocular pressure from secondary glaucoma prior to transplant, which can adversely affect both the success of subsequent corneal transplant as well as post-operative endothelial health. Early recognition and treatment of rejection or CMV reactivation are essential, with appropriate treatment instituted early. In spite of the above, late endothelial failure still occurred. Pathological alterations of aqueous humour with elevated aqueous cytokines have been postulated to contribute to endothelial cell loss after DSAEK [18]. The exact mechanism of corneal endotheliitis is still unknown, but autoimmune processes play an important role in the pathophysiology of CMV endotheliitis, with anterior chamber-associated immune deviation stimulated during intermittent reactivation of the CMV virus [19].

Endothelial keratoplasty has largely overtaken penetrating keratoplasty (PK) as the treatment of choice for endothelial disease, including CMV endotheliitis due to its superior safety profile, as well as more rapid and predictable visual outcomes as compared to PK. Many of the disadvantages of PK, such as suture-related complications, graft rejection and wound dehiscence, are also greatly reduced in DSAEK [20].

In summary, we illustrate the management of a patient who underwent endothelial keratoplasty for endothelial failure secondary to CMV endotheliitis. Targeted aqueous sampling for CMV-DNA titres preoperatively and help optimise graft timing and postoperatively to guide treatment. This ensures the adequacy of treatment and minimises the duration of systemic valganciclovir therapy to reduce the adverse effects of long-term treatment.

\section{Abbreviations}

BCVA: Best-corrected visual acuity; CMV: Cytomegalovirus; DSAEK: Descemet stripping automated endothelial keratoplasty; IOP: Intraocular pressure; KPs: Keratic precipitates; PCR: Polymerase chain reaction

\section{Acknowledgements}

Not applicable.

\section{Authors' contributions}

$M C$ and $L L$ reviewed the literature, collected the data and wrote the main manuscript text. DT, CSP and LL provided ophthalmic care to the patient. All authors participated in the critical revision and correction of the manuscript. All authors read and approved the final manuscript.

\section{Funding}

There was no funding support.

\section{Competing interest}

All authors have no conflicts of interest to disclose.

Ethics approval and consent to participate

Not applicable.

\section{Consent for publication}

Consent to publish the case report has been taken from the patient concerned and does not disclose the identity or infringe the privacy of the patient.

\section{Competing interests}

The authors declare that they have no competing interests.

\section{Author details}

${ }^{1}$ Singapore National Eye Centre, 11 Third Hospital Ave, Singapore, Singapore 168751, Singapore. ${ }^{2}$ Singapore Eye Research Institute, Singapore, Singapore. ${ }^{3}$ Department of Ophthalmology, Yong Loo Lin School of Medicine, National University of Singapore, Singapore, Singapore. ${ }^{4}$ Duke-NUS Medical School, Singapore, Singapore.

Received: 1 May 2019 Accepted: 19 July 2019

Published online: 02 August 2019

\section{References}

1. Khodadoust AA, Attarzadeh A (1982) Presumed autoimmune corneal endotheliopathy. Am J Ophthalmol 93:718-722

2. Chee S-P, Bacsal K, Jap A et al (2007) Corneal endotheliitis associated with evidence of cytomegalovirus infection. Ophthalmology 114:798-803. https://doi.org/10.1016/j.ophtha.2006.07.057

3. Koizumi N, Suzuki T, Uno T et al (2008) Cytomegalovirus as an etiologic factor in corneal endotheliitis. Ophthalmology 115:292-297.e3. https://doi. org/10.1016/j.ophtha.2007.04.053

4. Ang M, Sng CCA, Chee S-P et al (2013) Outcomes of corneal transplantation for irreversible corneal decompensation secondary to corneal endotheliitis in Asian eyes. Am J Ophthalmol 156:260-266.e2. https://doi.org/10.1016/j. ajo.2013.03.020

5. Cannon MJ, Schmid DS, Hyde TB (2010) Review of cytomegalovirus seroprevalence and demographic characteristics associated with infection. Rev Med Virol 20:202-213. https://doi.org/10.1002/rmv.655

6. Wong A, Tan KH, Tee CS, Yeo GS (2000) Seroprevalence of cytomegalovirus, toxoplasma and parvovirus in pregnancy. Singapore Med J 41:151-155

7. Koizumi N, Inatomi T, Suzuki T et al (2015) Clinical features and management of cytomegalovirus corneal endotheliitis: analysis of 106 cases from the Japan corneal endotheliitis study. British Journal of Ophthalmology 99:54-58. https://doi.org/10.1136/bjophthalmol-2013-3 04625

8. van Boxtel LAA, van der Lelij A, van der Meer J, Los LI (2007) Cytomegalovirus as a cause of anterior uveitis in immunocompetent patients. Ophthalmology 114:1358-1362. https://doi.org/10.1016/j.ophtha.2 006.09.035

9. Choi JA, Kim KS, Jung Y et al (2016) Cytomegalovirus as a cause of hypertensive anterior uveitis in immunocompetent patients. J Ophthalmic Inflamm Infect 6. https://doi.org/10.1186/s12348-016-0100-5

10. Anshu A, Chee S-P, Mehta JS, Tan DTH (2009) Cytomegalovirus endotheliitis in Descemet's stripping endothelial keratoplasty. Ophthalmology 116:624630. https://doi.org/10.1016/j.ophtha.2008.10.031

11. Agrawal R, Murthy S, Ganesh SK et al (2012) Cataract surgery in uveitis. Int J Inflam 2012(548453). https://doi.org/10.1155/2012/548453

12. Chee S-P, Jap A (2010) Cytomegalovirus anterior uveitis: outcome of treatment. Br J Ophthalmol 94:1648-1652. https://doi.org/10.1136/bjo.2 009.167767

13. Fellay J, Venetz J-P, Aubert J-D et al (2005) Treatment of cytomegalovirus infection or disease in solid organ transplant recipients with valganciclovir. Transplant Proc 37:949-951. https://doi.org/10.1016/j. transproceed.2004.11.066

14. Wong JXH, Agrawal R, Wong EPY, Teoh SC (2016) Efficacy and safety of topical ganciclovir in the management of cytomegalovirus (CMV)-related anterior uveitis. J Ophthalmic Inflamm Infect 6. https://doi.org/10.1186/ s12348-016-0078-z 
15. Helbig H, Noske W, Kleineidam M et al (1995) Bacterial endophthalmitis after anterior chamber paracentesis. Br J Ophthalmol 79:866

16. Trivedi D, Denniston AKO, Murray PI (2011) Safety profile of anterior chamber paracentesis performed at the slit lamp. Clin Experiment Ophthalmol 39:725-728. https://doi.org/10.1111/j.1442-9071.2011.02565.x

17. Van der Lelij A, Rothova A (1997) Diagnostic anterior chamber paracentesis in uveitis: a safe procedure. $\mathrm{Br} J$ Ophthalmol 81:976-979

18. Yazu H, Yamaguchi T, Aketa N et al (2018) Preoperative aqueous cytokine levels are associated with endothelial cell loss after Descemet's stripping automated endothelial keratoplasty. Invest Ophthalmol Vis Sci 59:612-620. https://doi.org/10.1167/iovs.17-23049

19. Suzuki T, Ohashi Y (2008) Corneal endotheliitis. Semin Ophthalmol 23:235240. https://doi.org/10.1080/08820530802111010

20. Tan DTH, Dart JKG, Holland EJ, Kinoshita S (2012) Corneal transplantation. Lancet 379:1749-1761. https://doi.org/10.1016/50140-6736(12)60437-1

\section{Publisher's Note}

Springer Nature remains neutral with regard to jurisdictional claims in published maps and institutional affiliations.

\section{Submit your manuscript to a SpringerOpen ${ }^{\circ}$ journal and benefit from:}

- Convenient online submission

- Rigorous peer review

- Open access: articles freely available online

High visibility within the field

- Retaining the copyright to your article

Submit your next manuscript at $\boldsymbol{\nabla}$ springeropen.com 